\title{
Structure of a glycosylphosphatidylinositol-anchored domain from a trypanosome variant surface glycoprotein.
}

\begin{abstract}
The cell surface of African trypanosomes is covered by a densely packed monolayer of a single protein, the variant surface glycoprotein (VSG). The VSG protects the trypanosome cell surface from effector molecules of the host immune system and is the mediator of antigenic variation. The sequence divergence between VSGs that is necessary for antigenic variation can only occur within the constraints imposed by the structural features necessary to form the monolayer barrier. Here, the structures of the two domains that together comprise the C-terminal di-domain of VSG ILTat1.24 have been determined. The first domain has a structure similar to the single C-terminal domain of VSG MITat1.2 and provides proof of structural conservation in VSG C-terminal domains complementing the conservation of structure present in the N-terminal domain. The second domain, although based on the same fold, is a minimized version missing several structural features. The structure of the second domain contains the C-terminal residue that in the native VSG is attached to a glycosylphosphatidylinositol (GPI) anchor that retains the VSG on the external face of the plasma membrane. The solution structures of this domain and a VSG GPI glycan have been combined to produce the first structure-based model of a GPI-anchored protein. The model suggests that the core glycan of the GPI anchor lies in a groove on the surface of the domain and that there is a close association between the GPI glycan and protein. More widely, the GPI glycan may be an integral part of the structure of other GPI-anchored proteins.
\end{abstract}

Keyword: Trypanosome; Variant surfaceglycoprotein; Trypanosoma brucei. 